\title{
Long non-coding RNA XIST inhibits osteoblast differentiation and promotes osteoporosis via Nrf2 hyperactivation by targeting CUL3
}

\author{
${\text { XIAO } \mathrm{CHEN}^{1}{ }^{1}, \text { FENGYU MA }^{2}, \text { NING ZHAI }^{3}, \text { FENG GAO }^{1} \text { and GUIJUN CAO }}^{1}$ \\ ${ }^{1}$ Department of Spinal Surgery, Affiliated Hospital of Jining Medical University, Jining, Shandong 272029; \\ ${ }^{2}$ Department of Spine Surgery, People's Hospital of Rizhao, Rizhao, Shandong 276800; \\ ${ }^{3}$ Imaging Department, Affiliated Hospital of Jining Medical University, Jining, Shandong 272029, P.R. China
}

Received December 3, 2020; Accepted March 26, 2021

DOI: $10.3892 /$ ijmm.2021.4970

\begin{abstract}
Osteoporosis (OP) is a common skeletal disorder characterized by a low bone mass and the deterioration of bone structure. Long non-coding (lnc)RNA X inactive-specific transcript (XIST) is highly expressed in the serum and monocytes of patients with OP. Thus, the purpose of the present study was to explore the mechanisms underlying the role of XIST in the progression of OP. To establish animal models of OP, female rats underwent a bilateral ovariectomy. The bone mineral density of individual rats was measured using dual-energy X-ray absorptiometry. The combination of XIST and cullin-3 (CUL3) was analyzed using a dual-luciferase reporter assay. Bone histopathological changes were assessed by hematoxylin and eosin staining. Alkaline phosphatase activity was examined by ALP staining. Finally, a series of functional experiments were performed to examine the effects of XIST on cellular behaviors. In the present study, XIST promoted $\mathrm{OP}$ and inhibited bone formation by regulating the expression levels of CUL3 and nuclear factor erythroid 2-related factor 2 (Nrf2) in the rats with OP. Moreover, XIST directly targeted CUL3 and negatively regulated its expression. Of note, CUL3 downregulation reversed the effects of XIST silencing on cell viability, differentiation and mineralization, as well as the expression of Nrf2 and CUL3 in MC3T3-E1 cells. Collectively, XIST was demonstrated to inhibit the differentiation of osteoblasts and promote OP by inhibiting the degradation of $\mathrm{Nrf} 2$ via targeting CUL3.
\end{abstract}

Correspondence to: Dr Guijun Cao, Department of Spinal Surgery, Affiliated Hospital of Jining Medical University, 89 Guhuai Road, Jining, Shandong 272029, P.R. China

E-mail: caoguijun0406@126.com

Key words: long non-coding RNA $\mathrm{X}$ inactive-specific transcript, osteoblasts, differentiation, nuclear factor erythroid 2-related factor 2, osteoporosis

\section{Introduction}

Osteoporosis (OP) is a common skeletal disorder characterized by a low bone mass, the deterioration of bone tissue and damaged bone structure (1). OP can increase the risk of fractures and other severe bone-related diseases, leading to increased mortality and healthcare costs worldwide $(2,3)$. Importantly, an increased number of OP-induced fracture cases occur in females aged $>55$ years (2). Accumulating evidence now suggests that $\mathrm{OP}$ in postmenopausal females is a commonly encountered skeletal disease, which is responsible for the increased risk of disability in thousands of individuals (4). Various complex signaling pathways are involved in the process of OP, and the exact molecular mechanisms responsible for the pathogenesis of OP remain unclear.

A previous study reported that osteoblasts and osteoclasts mediate bone formation and resorption in the process of bone remodeling, which is vital for the maintenance of bone homeostasis (5). Accumulating evidence has indicated that reactive oxygen species (ROS) play a key role in regulating bone homeostasis. An earlier study reported that the overproduction of ROS, induced by estrogen deficiency, suppressed osteoblast differentiation in vitro (6). Moreover, increased oxidative stress can cause bone loss by modulating the differentiation and survival of osteoblasts in vivo (7). As a transcription factor, nuclear factor erythroid 2-related factor 2 (Nrf2) can exert its cytoprotective effects under conditions of oxidative stress (8). It has been reported that Nrf2 exerts suppressive effects on bone resorption and subsequently promotes bone formation (9). However, Nrf2 overexpression has been demonstrated to be negatively associated with the differentiation of osteoblastic MC3T3-E1 cells (10). In addition, Nrf2 hyperactivation has been reported to be responsible for the impaired differentiation of osteoblasts (11). Hence, the biological role of Nrf2 in bone development should be further elucidated.

It was previously reported that the kelch-like ECH-associated protein 1 (Keap1)/cullin-3 (CUL3)/E3 ubiquitin-protein ligase RBX1 (RBX1) complex is a negative regulator of the Nrf2 protein (12). When any component of the complex is disrupted, the function of the Keap1/CUL3/RBX1 complex can become dysregulated, thus inducing Nrf2 hyperactivation. Of note, the present study discovered the interaction between CUL3 and long non-coding RNA (lncRNA) X 
inactive-specific transcript (XIST) on the Starbase website (http://starbase.sysu.edu.cn/index.php). IncRNAs are a type of endogenous non-coding RNA with a transcriptional length of >200 nucleotides (13). IncRNAs exert a regulatory effect on gene expression and are involved in various physiological processes, including nuclear transport, epigenetics, as well as transcriptional and post-transcriptional regulation (14). Among these lncRNAs, IncRNA XIST is highly expressed in the serum and monocytes of patients with OP, suggesting that lncRNA XIST promotes OP development (15). Furthermore, XIST knockdown exerts suppressive effects on the progression of tumors by reducing cell proliferation, invasion and migration, and promoting the apoptosis of human glioblastoma stem cells (16). Thus, in the present study, it was hypothesized that XIST was associated with the pathogenesis of OP via the regulation of Nrf2 expression by targeting CUL3.

In the present study, rats underwent ovariectomy (OVX) to construct an animal model of OP, in order to investigate whether XIST inhibits the differentiation of osteoblasts and promotes OP, and elucidate the role of Nrf2 in this process, in order to determine whether XIST may serve as an effective therapeutic candidate for OP.

\section{Materials and methods}

Animals and rat model of OP. A total of 78 female Wistar rats, aged 8 weeks and weighing 240-250 g, were obtained from Oriental Bioservice, Inc. These rats were acclimated for 1 week prior to the start of the study. The animals were housed in a temperature-controlled room $\left(22 \pm 2^{\circ} \mathrm{C}\right)$ with a 12 -h light/dark cycle and $55 \pm 10 \%$ relative humidity. The animals were fed a standard chow diet and had access to water ad libitum. Following anesthesia with isoflurane (induction dose, $4 \%$; maintenance dose, $2.5 \%$ ), the rats underwent bilateral OVX, as previously described $(17,18)$. In breif, an incision was made in the middle of the abdomen into the abdominal cavity under aseptic conditions following anesthesia, the bilateral ovaries were removed, and the same amount of greater omentum was removed in the sham-operated group. The animal surgical procedures were performed in accordance with the Guidelines for Animal Care and were approved by the Ethics Committee at the Affiliated Hospital of Jining Medical University (Jining, China, No. IACUC-2000509-5). All operations were carried out under sterile conditions in the specific pathogen-free animal center. Firstly, the animals were randomly assigned into the following six groups to examine the efficiency of transfection $(n=3)$ : i) The control group [injection of the same volume of the vehicle control (PBS) into knee joints] $(19,20)$; ii) shRNA-NC group (injection of shRNA-NC into knee joints); iii) shRNA-XIST-1 group (injection of shRNA-XIST-1 into knee joints); iv) shRNA-XIST-2 group (injection of shRNA-XIST-2 into knee joints); v) OverExp-NC group (injection of overexpression-NC plasmids into knee joints); and vi) OverExp-XIST group (injection of overexpression-XIST plasmids into knee joints). Subsequently, the animals (different rats) were randomly assigned into the following five groups using simple randomization $(n=12)$ : i) The control group [sham operation + injection of the same volume of vehicle control (PBS) into knee joints]; ii) overexpression (OverExp)-XIST group control (sham operation + injection of overexpression-XIST plasmids into knee joints); iii) model group (OVX + injection of the same volume of control vehicle (PBS) into knee joints); iv) model + short hairpin (sh)RNA-negative control (NC) group (OVX + injection of shRNA-NC into knee joints); and v) model + shRNA-XIST group (OVX + injection of shRNA-XIST into knee joints). The intra-articular injection of $50 \mu \mathrm{l}$ lentivirus or PBS was performed every 2 days for 10 weeks post-surgery, and the animals administered buprenorphine $(0.05 \mathrm{mg} / \mathrm{kg}$, s.c.) daily and were sacrificed in two batches at 10 and 12 weeks post-surgery. All rats were rapidly euthanized by cervical dislocation during deep anesthesia, induced by an intraperitoneal (i.p.) injection of $3.6 \%$ chloral hydrate solution at a concentration of $360 \mathrm{mg} / \mathrm{kg}$ at the end of the experiment. The femurs were harvested for histological analysis and RNA extraction.

Cell culture and treatment. The murine osteoblastic cell line MC3T3-E1 (ATCC ${ }^{\circledR}$ CRL-2594) was obtained from the American Type Culture Collection. Cells were maintained in $\alpha$-minimal essential medium ( $\alpha$-MEM) containing $10 \%$ fetal bovine serum (Gibco; Thermo Fisher Scientific, Inc.), $100 \mathrm{U} / \mathrm{ml}$ penicillin and $100 \mu \mathrm{g} / \mathrm{ml}$ streptomycin (Gibco; Thermo Fisher Scientific, Inc.) at $37^{\circ} \mathrm{C}$ in a humid environment with $5 \% \mathrm{CO}_{2}$, and the medium was replaced every 2 days. When cell confluency reached 80-90\%, MC3T3-E1 cells were cultured in differentiation induction medium, which was complete medium including L-ascorbic acid $(50 \mu \mathrm{g} / \mathrm{ml})$ and $\beta$-glycerophosphate $(5 \mathrm{mM})$. Finally, the MC3T3-E1 cells were exposed to $0.3 \mathrm{mM}$ hydrogen peroxide $\left(\mathrm{H}_{2} \mathrm{O}_{2}\right)$ for $24 \mathrm{~h}$ to induce dysfunction and oxidative stress in vitro.

Transfection. The interaction between CUL3 and IncRNA $\mathrm{X}$ inactive-specific transcript (XIST) was predicted with the Starbase website. After the cell confluency reached $80 \%$, MC3T3-E1 cells were serum-starved for $3 \mathrm{~h}$, and then transfected with shRNA-XIST (50 nM, shRNA-XIST-1, 5'-GCT GACTACCTGAGATTTAAG-3'; shRNA-XIST-2, 5'-GCT CTTGAACAGTTAATTTGC-3'), OverExp-XIST (100 nM), shRNA-CUL3 (50 nM, shRNA-CUL3-1, 5'-GCGAGAAGA TGTACTAAATTC-3'; shRNA-CUL3-2, 5'-GCTTGGAAT GATCATCAAACA-3') or the shRNA-NC, (5'-TTCTCC GAACGTGTCACGT-3') using Lipofectamine ${ }^{\circledR} 2000$ reagent (Invitrogen; Thermo Fisher Scientific, Inc.), respectively. XIST-overexpression plasmids and control overexpression plasmids were cloned into a pCDNA3.1 vector and provided by Shanghai GenePharma Co., Ltd. Following $6 \mathrm{~h}$ of incubation at $37^{\circ} \mathrm{C}$, the serum-free medium was exchanged for medium with serum. Finally, the MC3T3-E1 cells were cultured for a further $24 \mathrm{~h}$ at $37^{\circ} \mathrm{C}$ and then used for subsequent experiments. The transfection efficiency was examined by reverse transcription-quantitative PCR (RT-qPCR).

Hematoxylin and eosin $(H \& E)$ staining. Distal femurs were collected and fixed in $10 \%$ neutral formaldehyde for $72 \mathrm{~h}$. Subsequently, the femur bones were removed and decalcified in $10 \%$ ethylene diamine tetraacetate solution $(\mathrm{pH} \mathrm{7.4)}$ for 3 weeks and embedded in paraffin, after which time they were cut into $5-\mu \mathrm{m}$-thick sections, stained with H\&E, and the field of interest at the distal femur was visualized under a fluorescence 
microscope (Olympus IX53; Olympus Corporation) at x200 magnification. The relative injury levels of each group were measured by the cortical area and analyzed using Image J software (version 1.48; National Institutes of Health).

Dual-energy X-ray absorptiometry (DXA) analysis. DXA measurement with Hologic DXA equipment (Hologic Discovery W 81507; Hologic, Inc.) was carried out to scan and measure the bone mineral density (BMD) of each individual rat. Results were expressed as the grams of mineral content per square centimeter of bone area $\left(\mathrm{g} / \mathrm{cm}^{2}\right)$.

Alkaline phosphatase (ALP) staining. Distal femurs were fixed with $80 \%$ alcohol and cut into $5-\mu$ m-thick sections using a cryostat. In addition, MC3T3-E1 cells $\left(1.2 \times 10^{4}\right.$ cells/well) were seeded in 6 -well plates. When the cells were at $80 \%$ confluency, they were treated with $\mathrm{H}_{2} \mathrm{O}_{2}$, or transfected as aforementioned in $\alpha$-MEM for 10 days. The determination of ALP activity was performed using ALP staining kits (Thermo Fisher Scientific, Inc.) both in vitro and in vivo (Roche Diagnostics $\mathrm{GmbH}$ ) according to the manufacturer's protocols. Subsequently, the OD value was measured at $540 \mathrm{~nm}$ using a microplate reader (Infinite ${ }^{\mathrm{TM}}$ M2000; Tecan Group, Ltd.), and the images were visualized and collected under an inverted fluorescence microscope (Olympus Corporation) at x200 magnification.

$R T$ - $q P C R$. Total RNA was extracted using TRIzol ${ }^{\circledR}$ reagent (Invitrogen; Thermo Fisher Scientific, Inc.), and the Reverse Transcription System kit (Takara Bio, Inc.) was used to generate cDNA. Subsequently, RT-qPCR was performed with an SYBR Premix Ex Taq kit (Takara Bio, Inc.). Relative expression was calculated using the $2^{-\Delta \Delta C q}$ method (21) and normalized to GAPDH expression. The results are expressed as the relative ratio to the control. The thermocycling conditions were as follows: Pre-denaturation at $95^{\circ} \mathrm{C}$ for $5 \mathrm{~min}$, followed by 40 cycles at $95^{\circ} \mathrm{C}$ for $15 \mathrm{sec}, 60^{\circ} \mathrm{C}$ for $30 \mathrm{sec}$ and $72^{\circ} \mathrm{C}$ for $30 \mathrm{sec}$. The primer sequences included were as follows: XIST forward, 5'-TCAGCCCATCAGTCCAAG ATC-3' and reverse, 5'-CCTAGTTCAGGCCTGCTTTTC AT-3'; Keap-1 forward, 5'-CCA ACTTCCTCAAGGAGC AG-3' and reverse, 5'-CGGCGACAAATATCATCCTT-3'; Nrf2 forward, 5'-GGCTACGTTTCAGTCACTTG-3' and reverse, 5'-AACTCAGGA ATGGATAATAG-3'; CUL3 forward, 5'-GATGAGTTCAGGCAACATC-3' and reverse, 5'-ATGTCTTGGTGCTGGTGG-3'; ALP forward, 5'-GGA CCATTCCCACGTCTTCAC-3' and reverse, 5'-CCTTGTAGC CAGGCCCATTG-3'; bone morphogenetic protein 2 (BMP2) forward, 5'-CCCAGCGTGAAAAGAGAGAC-3' and reverse, 5'-GGAAGCAGCAACGCTAGAAG-3'; runt-related transcription factor 2 (Runx2) forward, 5'-TTACCCCTCCTACCT GAGCCAG-3' and reverse, 5'-GGTGTGGTAGTGAGTGGT GG-3'; osterix (OSX) forward, 5'-GCCTACTTACCGTGA CTTT-3' and reverse, 5'-GCCCACTATTGCCAACTGC-3'; GAPDH forward, 5'-AAGGTGAAGGTCGGAGTCAAC-3' and reverse, 5'-GGGGTCATTGATGGCAACAATA-3'.

Western blot analysis. Bone tissues and MC3T3-E1 cells were processed for protein extraction using RIPA lysis buffer (Takara Bio, Inc.), and then quantified using a BCA assay kit
(Bio-Rad Laboratories, Inc.). In brief, a total of $25 \mu \mathrm{g}$ protein samples were separated via 10\% SDS-PAGE, and separated proteins were then transferred onto PVDF membranes. After blocking with 5\% skimmed milk at room temperature for $2 \mathrm{~h}$, the membranes were incubated with primary antibodies against Keap-1 (1:1,000, ab227828, Abcam), CUL3 (1:1,000, ab75851, Abcam), Nrf2 (1:1,000, ab76026, Abcam), and GAPDH $\left(1: 2,000\right.$, ab8245, Abcam) overnight at $4^{\circ} \mathrm{C}$, and the membranes were then washed with PBS and incubated with the appropriate HRP-conjugated secondary antibody (1:5,000; cat. no. 7074; Cell Signaling Technology, Inc.) at room temperature for $2 \mathrm{~h}$. Finally, ECL solution was applied to bands to measure the protein expression levels with Tanon-5200 Chemiluminescence Imager (Tanon Science and Technology Co., Ltd.). The relative intensities of target proteins were analyzed using ImageJ software (version 1.48; National Institutes of Health) and normalized to GAPDH expression.

Cell Counting kit-8 (CCK-8) assay. CCK-8 assay was performed to determine cell viability. MC3T3-E1 cells were collected at $36 \mathrm{~h}$ following culture with CCK- 8 solution (Dojindo Molecular Technologies, Inc.). The absorbance of each sample at $450 \mathrm{~nm}$ was then measured using a microplate reader (Bio-Rad Laboratories, Inc.).

Flow cytometry. MC3T3-E1 cells were seeded in 6-well plates $\left(1 \times 10^{5}\right.$ cells/well) and subjected to specific transfection under $\mathrm{H}_{2} \mathrm{O}_{2}$ conditions. An Annexin V-FITC/PI apoptosis detection kit (556547, BD Biosciences) was used to evaluate the cell apoptotic rate. Cells were resuspended with $1 \mathrm{X}$ Binding Buffer (500 $\mu \mathrm{l})$, and incubated with $5 \mu \mathrm{l}$ Annexin V-FITC (5 $\mu \mathrm{l})$ and PI staining solution $(10 \mu \mathrm{l})$ for $15 \mathrm{~min}$ at room temperature in the dark. Following incubation in the dark for $30 \mathrm{~min}$ at room temperature, apoptosis was evaluated using a flow cytometer (BD Biosciences) with BD CellQuest Pro software version 5.2.1 (BD Biosciences).

Alizarin Red staining. Following incubation for $24 \mathrm{~h}$ at $37^{\circ} \mathrm{C}$, MC3T3-E1 cells were fixed with $4 \%$ paraformaldehyde for $15 \mathrm{~min}$ at room temperature and rinsed with $\mathrm{dd}_{2} \mathrm{O}$. Subsequently, $1 \%$ Alizarin Red solution was prepared and the $\mathrm{pH}$ was adjusted to 4.2 . Subsequently, the cells were stained with Alizarin Red staining solution for $15 \mathrm{~min}$ at room temperature and captured under an inverted fluorescence microscope (Olympus IX53; Olympus Corporation).

Dual-luciferase reporter assay. The combination of XIST and CUL3 was evaluated by performing a dual-luciferase reporter assay. Briefly, MC3T3-E1 cells were seeded in 6-well plates and differentiation was induced for $24 \mathrm{~h}$ after the cell confluency reached $80 \%$. The cells were then co-transfected with pmirGLO vector (Promega Corporation) recombined with wild-type (WT, with the binding sites in XIST)/mutant-type (MUT, with the mutant binding sites in XIST) and WT/MUT 3'UTR of CUL3 (CUL3-WT and CUL3-MUT) using Lipofectamine ${ }^{\circledR} 2000$. Following $24 \mathrm{~h}$ of transfection, cells were collected and the Firefly luciferase activity was quantified using the Dual-Luciferase Reporter Assay System (Promega Corporation). The data were normalized to Renilla luciferase activity. 
Statistical analysis. All data were obtained from three independent experiments and are expressed as the mean \pm SEM. Differences between groups were statistically analyzed with an unpaired Student's t-test or one-way ANOVA followed by Tukey's post hoc test. All statistical analyses were performed with the GraphPad Prism 5 software package (GraphPad Software, Inc.). $\mathrm{P}<0.05$ was considered to indicate a statistically significant difference.

\section{Results}

XIST promotes $O P$ and inhibits bone formation in rats with $O V X$-induced $O P$. First, the shRNA-XIST and OverExp-XIST plasmids were constructed to regulate the expression of XIST. As shown in Fig. 1, shRNA-XIST-1 exerted a more significant knockdown effect on XIST expression than shRNA-XIST-2. Thus, shRNA-XIST-1 was selected for use in further experiments. In addition, OverExp-XIST plasmids notably enhanced the expression of XIST. The results of RT-qPCR suggested that these plasmids could be used for subsequent experiments due to their high transfection efficiencies. Second, OverExp-XIST and shRNA-XIST plasmids were injected into the knee joints of rats to examine the effects of XIST on OP pathogenesis. As demonstrated by the results of DXA, the BMD of the rats from the OVX model group was decreased compared with that in the control group. Following treatment with OverExp-XIST and shRNA-XIST for 12 weeks, the BMD of the rats in the OverExp-XIST group was decreased compared with that in the control group, whereas the BMD of the rats in the model + shRNA-XIST group was increased compared with that in the model group (Fig. 2A), suggesting that XIST accelerated the progression of OP and thus aggravated the severity of OP.

To further examine the effects of XIST on the pathogenesis of OP, histopathological changes in bone and ALP activity were determined by H\&E and ALP staining, respectively. The results of $\mathrm{H} \& \mathrm{E}$ staining revealed that the OVX procedure led to a significant decrease in the cortical area in the rats in the model group. The cortical area of the OverExp-XIST rats was notably decreased compared with that of the control rats at the 12 th week, and thus the relative injury levels were increased. However, the cortical area of the rats in the model + shRNA-XIST group was significantly increased compared with that of the rats in the model group, with a decrease in the relative injury levels (Fig. 2B). Moreover, ALP is a known biomarker of bone formation; therefore, ALP staining was performed to confirm the role of XIST in the progression of OP. It was observed that ALP activity was inhibited by OVX and XIST overexpression, and the OVX-inhibited ALP activity was increased by XIST downregulation in vivo at the 12th week (Fig. 2C). Moreover, RT-qPCR revealed that the mRNA expression levels of osteoblastic differentiation makers (ALP, BMP2, RUNX2 and OSX) were suppressed by OVX and XIST overexpression, whereas these expression levels were elevated by XIST silencing (Fig. 2D). These results indicated that XIST aggravated OP and hampered bone formation in rats with OVX-induced OP.

XIST regulates the expression levels of CUL3 and Nrf2 in rats with $O V X$-induced $O P$. To examine the effects of XIST on Keap-1, CUL3 and Nrf2 expression, western blot analysis and RT-qPCR were carried out to assess the protein and mRNA

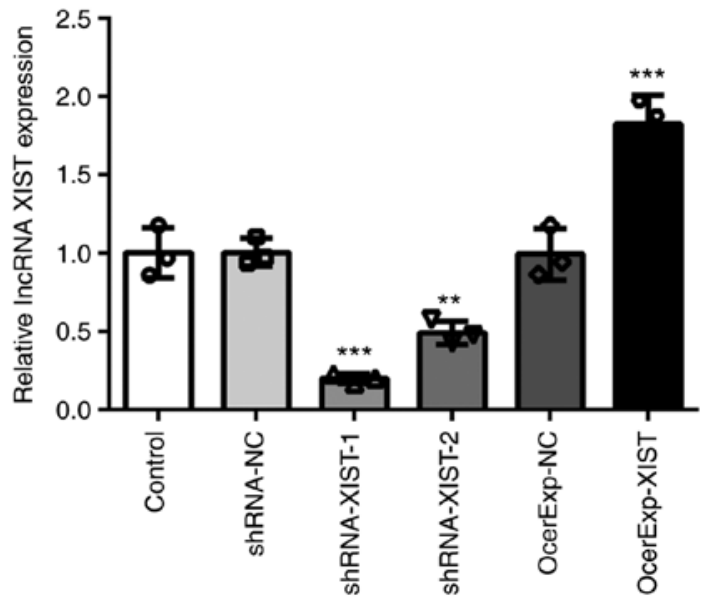

Figure 1. Relative mRNA expression of XIST. The relative expression of XIST in femurs was detected via reverse transcription-quantitative PCR. Results are presented as the mean \pm SEM from three independent experiments. ${ }^{* *} \mathrm{P}<0.01,{ }^{* * *} \mathrm{P}<0.001$ vs. Control. XIST, $\mathrm{X}$ inactive-specific transcript.

expression levels of Keap-1, CUL3 and Nrf2 in rats with OP, respectively. As shown in Fig. 3, the results of RT-qPCR revealed that OverExp-XIST transfection and OVX induced XIST overexpression, and shRNA-XIST led to XIST downregulation. Moreover, XIST overexpression led to a reduction in the expression of CUL3, and induced an upregulation in the expression of Nrf2. By contrast, XIST knockdown induced an upregulation in the expression of CUL3, and led to the downregulation of Nrf2 expression. Additionally, XIST did not exert any marked regulatory effects on the expression of Keap-1. As shown in Fig. 4, the results of western blot analysis demonstrated that XIST exerted similar effects on the protein expression of these genes. Of note, the ubiquitination of Nrf2 was inhibited by XIST overexpression, but promoted by XIST knockdown. These data suggested that XIST played a regulatory role in the expression of CUL3 and Nrf2.

XIST downregulation promotes the survival, differentiation and mineralization of $\mathrm{H}_{2} \mathrm{O}_{2}$-treated MC3T3-El cells. To confirm the role of XIST in OP pathogenesis, a series of functional assays were performed in vitro to determine the effects of XIST on the cellular behaviors of MC3T3-E1 cells. Firstly, shRNA-XIST and OverExp-XIST were transfected into the cells to achieve XIST silencing and overexpression, respectively. As the expression of XIST in the shRNA-XIST-1 group was lower than that in shRNA-XIST-2 group, shRNA-XIST-1 was selected for use in subsequent experiments, as demonstrated by the RT-qPCR results (Fig. 5A). MC3T3-E1 cells were exposed to $0.3 \mathrm{mM} \mathrm{H}_{2} \mathrm{O}_{2}$ to induce dysfunction and oxidative stress, which was defined as an in vitro OP cell model. The results of CCK- 8 assay revealed that $\mathrm{H}_{2} \mathrm{O}_{2}$ treatment significantly reduced the survival rate of the MC3T3-E1 cells, which was blocked by XIST silencing (Fig. 5B). Furthermore, the results of flow cytometry demonstrated that $\mathrm{H}_{2} \mathrm{O}_{2}$ treatment promoted cell apoptosis, whereas XIST knockdown attenuated the apoptotic rate of the MC3T3-E1 cells in the model group (Fig. 5C and D). The aforementioned data indicated that XIST knockdown improved the survival rate of MC3T3-E1 cells which was decreased by $\mathrm{H}_{2} \mathrm{O}_{2}$ treatment. 

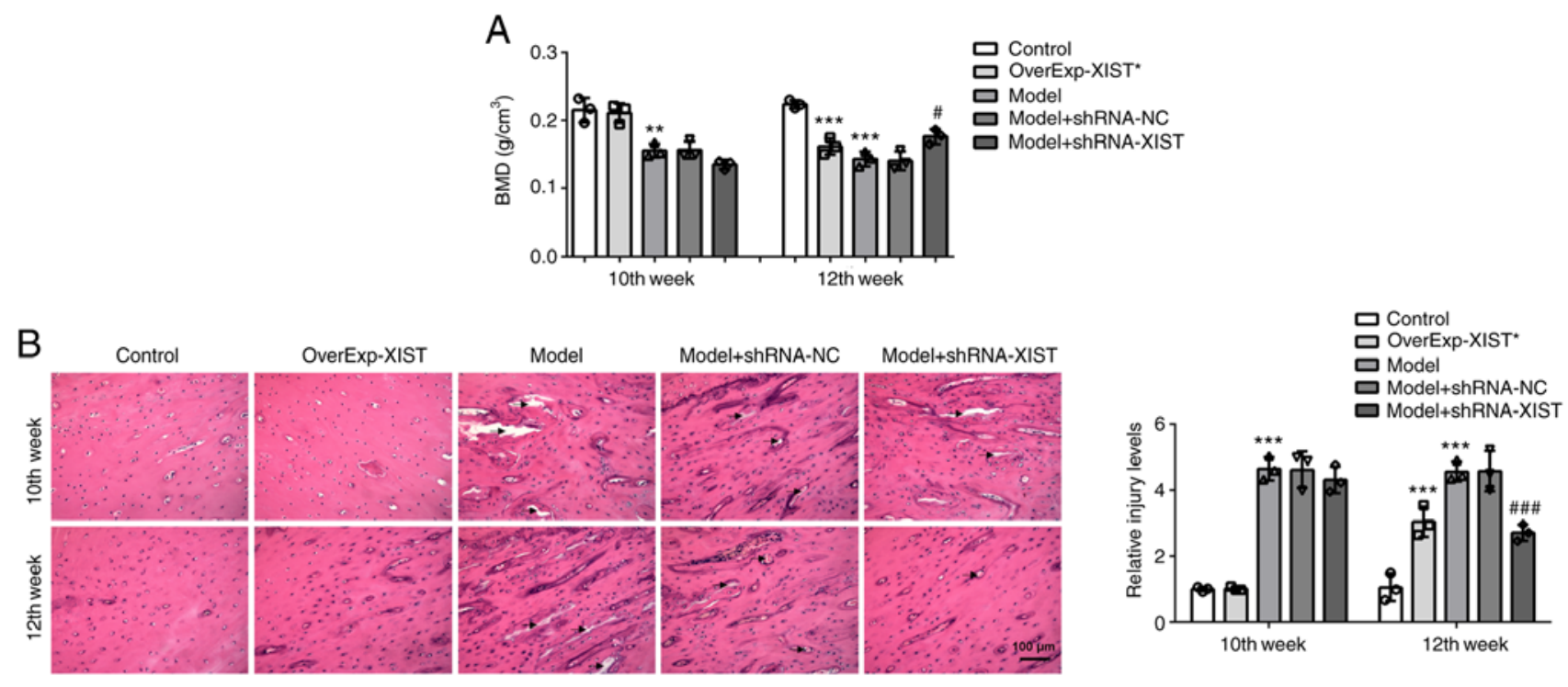

C
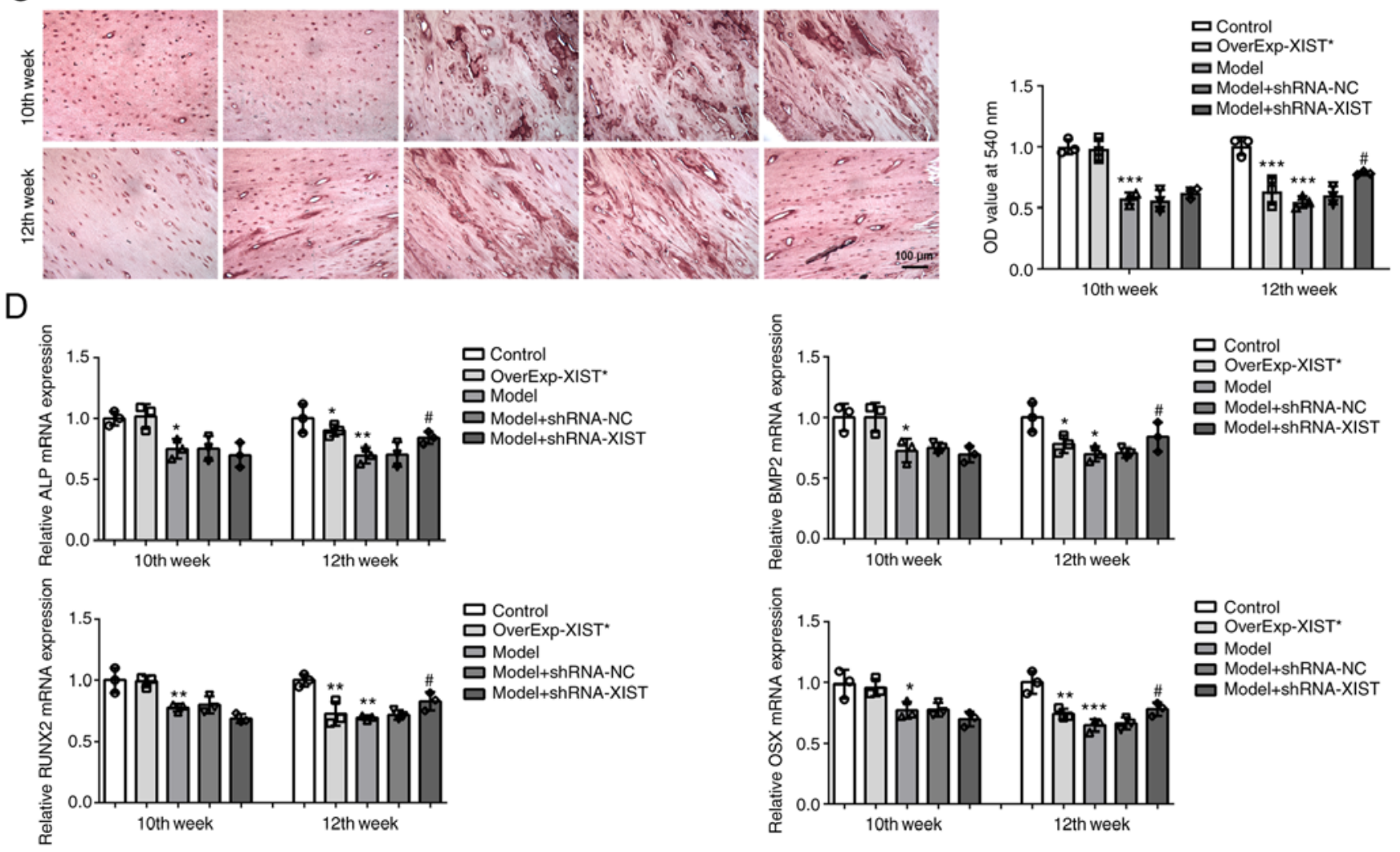

Figure 2. X inactive-specific transcript promotes OP and suppresses bone formation in the ovariectomy-induced OP rats. (A) Bone mineral density of rats from each group was measured via DXA with Hologic DXA equipment. (B) Bone histopathological changes in groups (Control, OverExp-XIST, Model, Model+shRNA-NC, Model+shRNA-XIST) were analyzed by hematoxylin and eosin staining. Scale bar, $100 \mu \mathrm{m}$. Arrows indicate the destructive area of the cortex. (C) ALP activity was detected by ALP staining. Scale bar, $100 \mu \mathrm{m}$. (D) The relative mRNA expression levels of ALP, bone morphogenetic protein 2, runt-related transcription factor 2 and osterix in femurs were detected via reverse transcription-quantitative PCR. Results are presented as the mean \pm SEM from three independent experiments. ${ }^{*} \mathrm{P}<0.05,{ }^{* *} \mathrm{P}<0.01,{ }^{* * *} \mathrm{P}<0.001$ vs. control; ${ }^{*} \mathrm{P}<0.05,{ }^{\# \# *} \mathrm{P}<0.01$ vs. model group. OP, osteoporosis; DXA, dual-energy X-ray absorptiometry; ALP, alkaline phosphatase.

Moreover, Alizarin Red staining and ALP assays determined the differentiation and mineralization abilities of MC3T3-E1 cells, respectively. The Alizarin Red staining results revealed that the number of calcium nodules was decreased following $\mathrm{H}_{2} \mathrm{O}_{2}$ treatment. However, XIST silencing significantly increased the number of calcium nodules that was reduced by $\mathrm{H}_{2} \mathrm{O}_{2}$ treatment (Fig. $5 \mathrm{E}$ and $\mathrm{F}$ ), suggesting that the knockdown of XIST expression enhanced the mineralization ability of MC3T3-E1 cells under oxidative stress conditions. Additionally, ALP assay indicated that MC3T3-E1 cells in the model group had a lower differentiation ability compared with the control group, and that XIST downregulation notably enhanced the differentiation ability of the cells compared with the model group (Fig. 5G). Finally, the results of RT-qPCR demonstrated that $\mathrm{H}_{2} \mathrm{O}_{2}$ treatment induced the upregulation of XIST and Nrf2 expression levels, and the downregulation 

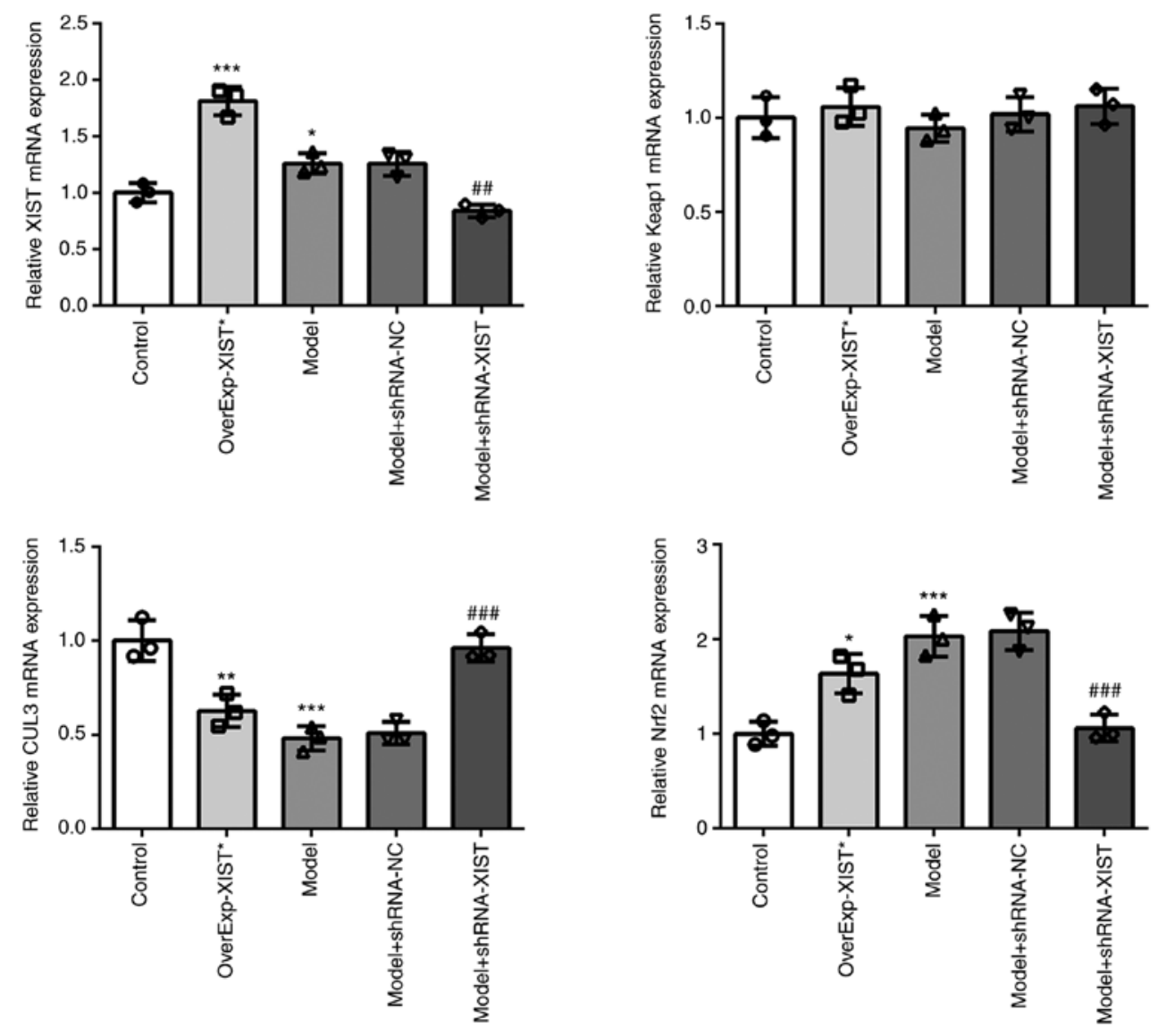

Figure 3. XIST modulates the mRNA expression levels of CUL3 and Nrf2 in the ovariectomy-induced osteoporosis rats. The relative expression levels of XIST, kelch-like ECH-associated protein 1, CUL3 and Nrf2 were detected via reverse transcription-quantitative PCR. Results are presented as the mean \pm SEM from three independent experiments. ${ }^{*} \mathrm{P}<0.05,{ }^{* *} \mathrm{P}<0.01,{ }^{* * * *} \mathrm{P}<0.001$ vs. control; ${ }^{\# \#} \mathrm{P}<0.01,{ }^{\# \# \#} \mathrm{P}<0.001$ vs. model group. XIST, X inactive-specific transcript; CUL3, cullin-3; Nrf2, nuclear factor erythroid 2-related factor 2.
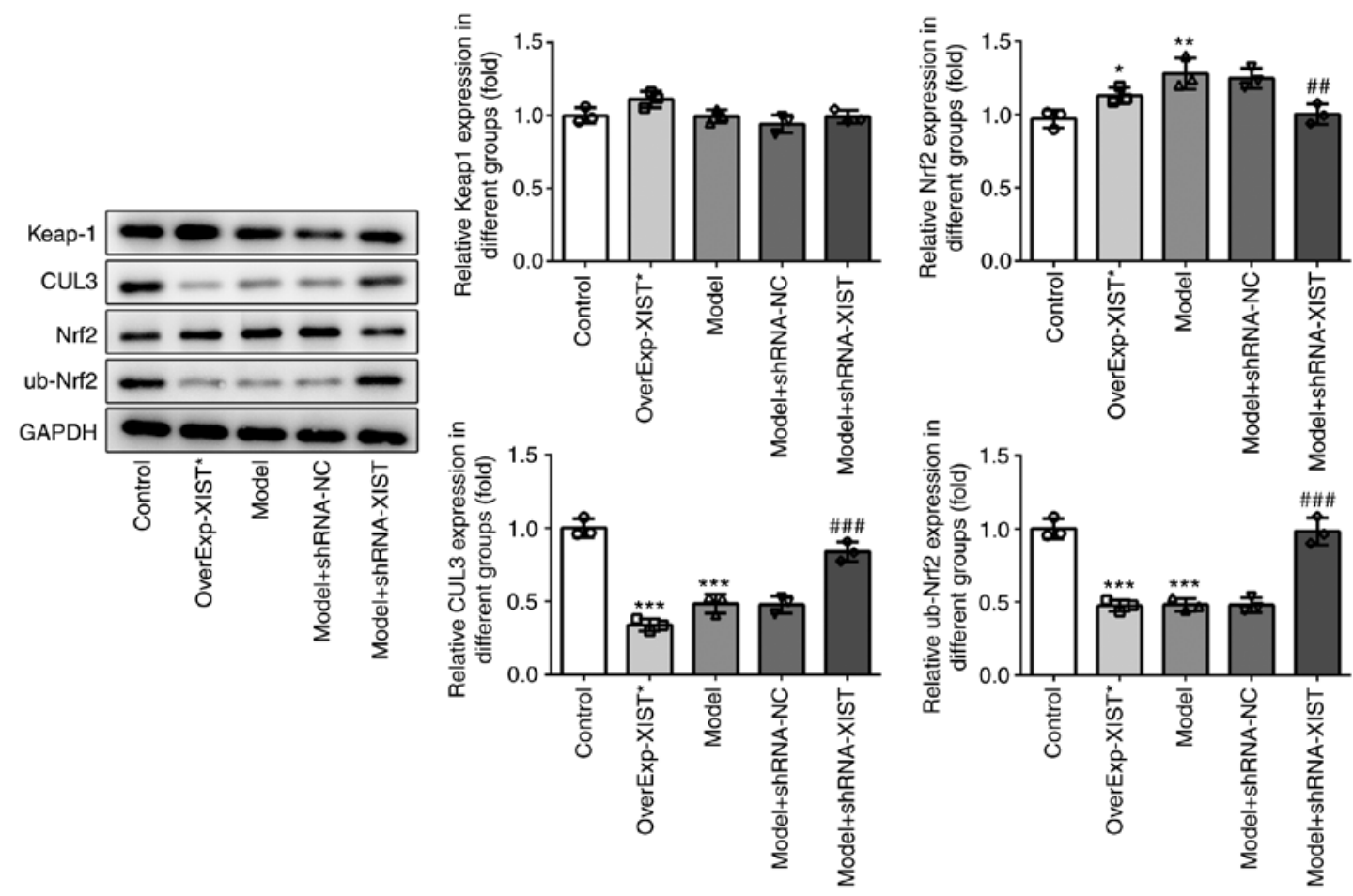

Figure 4. X inactive-specific transcript modulates the protein expression levels of CUL3 and Nrf2 in the ovariectomy-induced osteoporosis rats. The expression levels of kelch-like ECH-associated protein 1, CUL3, Nrf2 and ubiquitin Nrf2 were detected via western blot analysis. GAPDH was set as the internal normalization control. Results are presented as the mean \pm SEM from three independent experiments. ${ }^{*} \mathrm{P}<0.05,{ }^{* *} \mathrm{P}<0.01,{ }^{* * *} \mathrm{P}<0.001 \mathrm{vs}$. control; ${ }^{\# \#} \mathrm{P}<0.01$, ${ }^{\# \# \text { P }}<0.001$ vs. model group. CUL3, cullin-3; Nrf2, nuclear factor erythroid 2-related factor 2. 


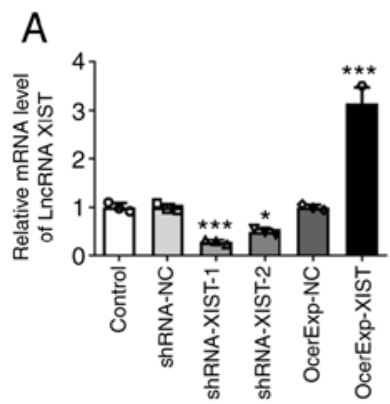

B

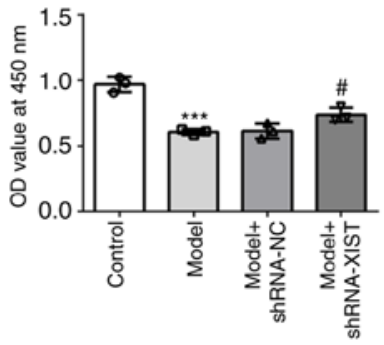

D

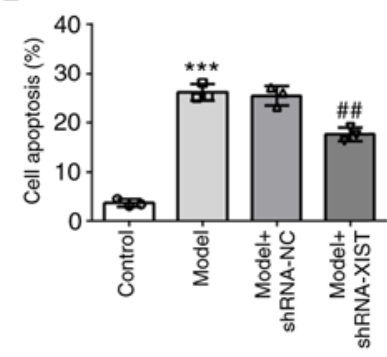

C
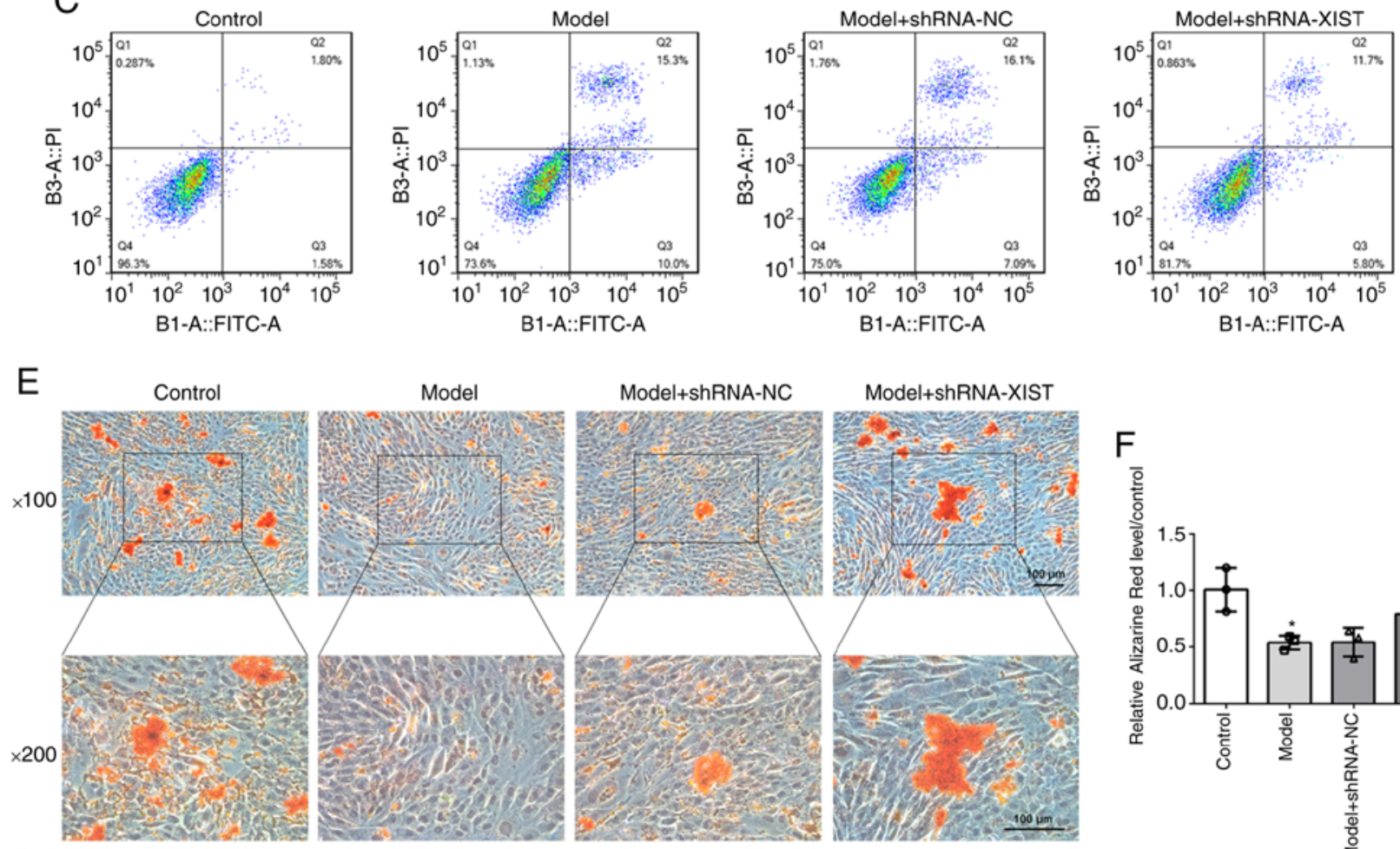

Model+shRNA-NC

Model+shRNA-XIST
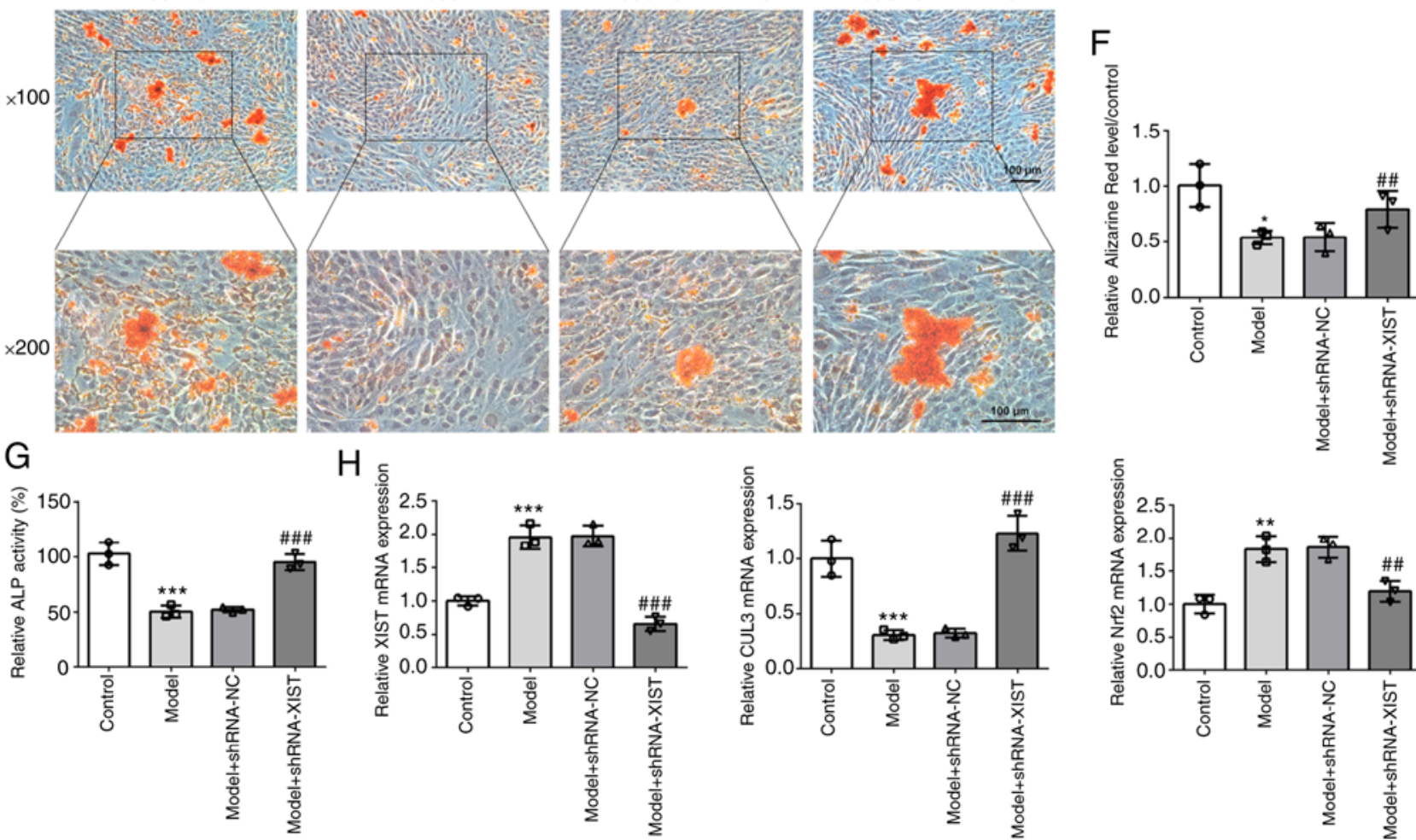

Figure 5. XIST knockdown improves the survival, differentiation and mineralization of cells, and alters mRNA levels of CUL3 and $\mathrm{Nrf}_{2}$ in $\mathrm{H}_{2} \mathrm{O}_{2}$-treated MC3T3-E1 cells. (A) The relative mRNA expression level of XIST was detected via reverse transcription-quantitative PCR. (B) The survival rate of cells treated with or without $\mathrm{H}_{2} \mathrm{O}_{2}$ was evaluated using a Cell Counting Kit-8 assay. (C and D) The cell apoptotic rate was determined via flow cytometry. (E and F) The mineralization ability of MC3T3-E1 cells was determined by Alizarin Red staining. Scale bar, $100 \mu \mathrm{m}$. (G) ALP activity was detected using an ALP assay kit. (H) The relative mRNA expression levels of XIST, CUL3 and Nrf2 were detected via reverse transcription-quantitative PCR. Results are presented as the mean \pm SEM from three independent experiments. ${ }^{*} \mathrm{P}<0.05,{ }^{* *} \mathrm{P}<0.01,{ }^{* * *} \mathrm{P}<0.001$ vs. control; ${ }^{\#} \mathrm{P}<0.05,{ }^{\# \#} \mathrm{P}<0.01,{ }^{\# \# \# /} \mathrm{P}<0.001$ vs. model group. $\mathrm{XIST}, \mathrm{X}$ inactive-specific transcript; CUL3, cullin-3; Nrf2, nuclear factor erythroid 2-related factor 2; ALP, alkaline phosphatase.

of CLU3 expression, which were reversed by the silencing of XIST (Fig. 5H).

Interaction between XIST and CUL3. Subsequently, the underlying mechanisms of XIST in OP pathogenesis were investigated. Herein, the interaction between XIST and CUL3 was confirmed using a dual-luciferase reporter assay. As shown in Fig. 6A and B, the luciferase activity was notably decreased in the MC3T3-E1 cells when the pmirGLO-CUL3-3'UTR WT plasmid was co-transfected with WT-XIST. No differences were found in other groups. These findings indicated that XIST directly targeted CUL3 and negatively modulated its expression. 
A
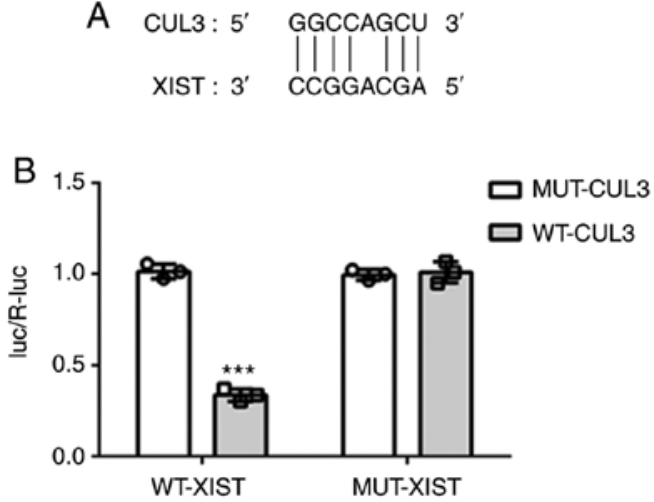

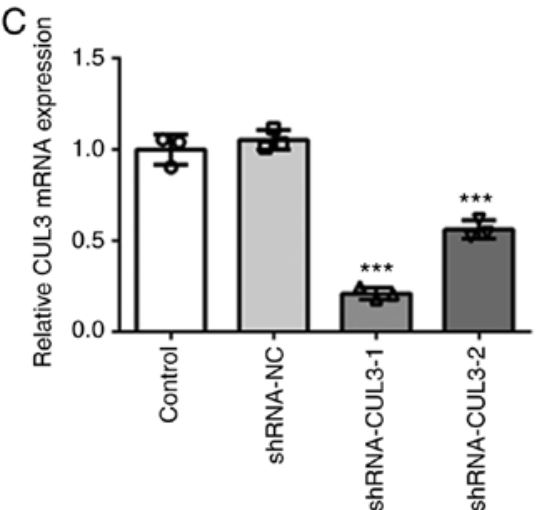

Figure 6. Interaction between XIST and CUL3. (A) 3'-untranslated region of CUL3 is partially complementary to XIST. (B) The interaction between XIST and CUL3 was analyzed via a dual-luciferase reporter assay. (C) The relative expression of CUL3 was detected via reverse transcription-quantitative PCR. Results are presented as the mean \pm SEM from three independent experiments. ${ }^{* * *} \mathrm{P}<0.001$ vs. control. XIST, X inactive-specific transcript; CUL3, cullin-3.

A

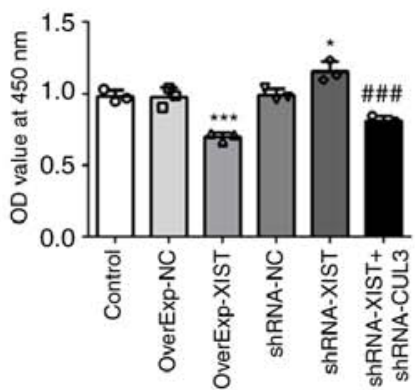

$\mathrm{B}$

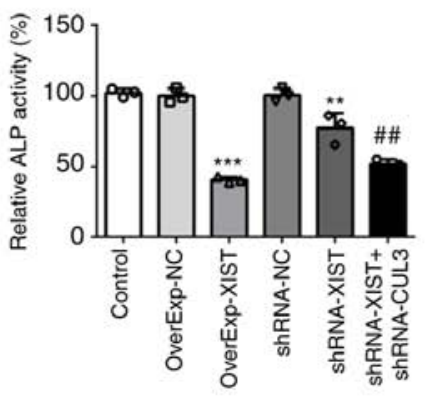

$\mathrm{D}$

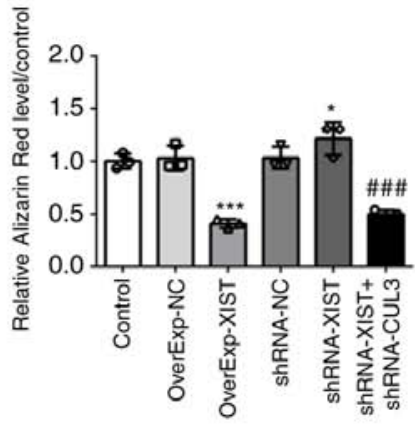

c

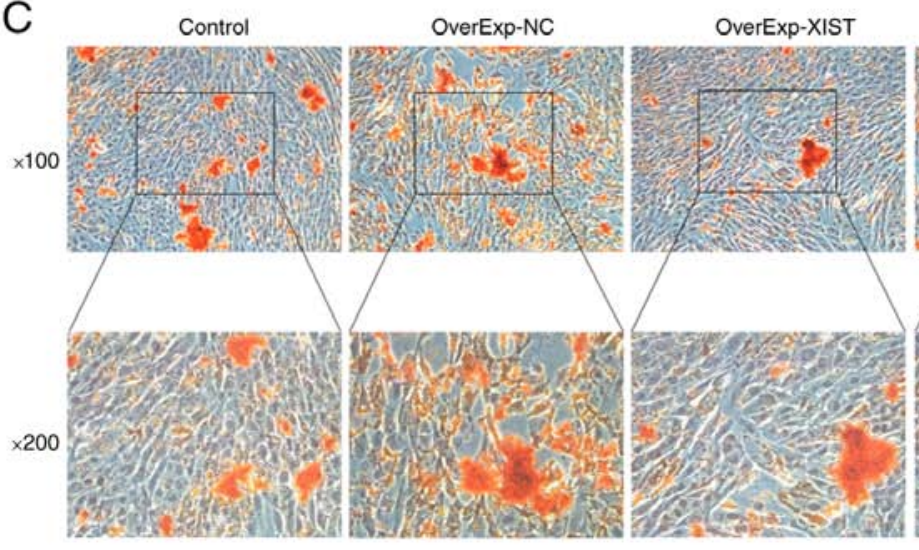

ShRNA-NC

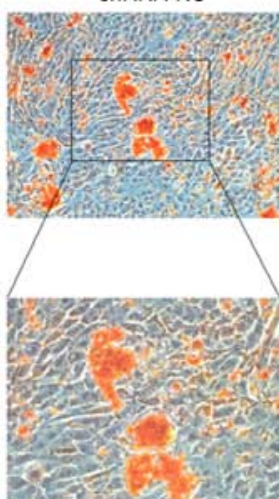

ShRNA-XIST

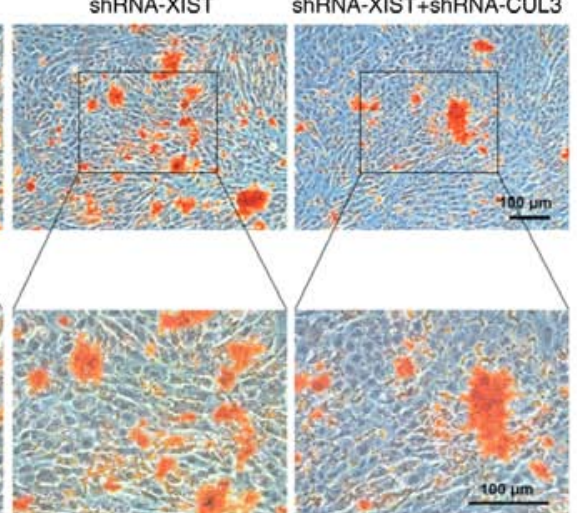

Figure 7. CUL3 downregulation reverses the XIST knockdown-induced effects on cell viability, differentiation and mineralization of MC3T3-E1 cells. (A) The survival rate of cells transfected with OverExp-NC, OverExp-XIST, shRNA-NC, shRNA-XIST or shRNA-XIST + shRNA-CUL3-1 was evaluated with a Cell Counting Kit-8 assay. (B) ALP activity was elevated, as determined using an ALP assay kit. (C and D) The mineralization ability of MC3T3-E1 cells was determined using Alizarin Red staining. Scale bar, $100 \mu \mathrm{m}$. Results are presented as the mean $\pm \mathrm{SEM}$ from three independent experiments. ${ }^{*}<0.05,{ }^{* *} \mathrm{P}<0.01$, ${ }^{* * * *} \mathrm{P}<0.001$ vs. control; ${ }^{\# \#} \mathrm{P}<0.01,{ }^{\# \#} \mathrm{P}<0.001$ vs. shRNA-XIST. XIST, X inactive-specific transcript; CUL3, cullin-3; ALP, alkaline phosphatase; OverExp, overexpression; NC, negative control; shRNA, short hairpin.

Downregulation of CUL3 reverses the effects of XIST silencing on cell viability, differentiation and mineralization, as well as the expression levels of Nrf2 and CUL3 in MC3T3-E1 cells. To knockdown the expression of CUL3, shRNA-CUL3 plasmids were constructed. As demonstrated by the results of RT-qPCR, the decrease in the expression of CUL3 in the shRNA-CUL3-1 group was significantly higher than that in the shRNA-CUL3-2 group (Fig. 6C). Therefore, shRNA-CUL3-1 plasmids were employed in subsequent experiments.

To confirm the CUL3-mediated role of XIST in OP progression, the cellular behaviors were observed when the
MC3T3-E1 cells were separately transfected with shRNA-NC, shRNA-XIST and shRNA-XIST + shRNA-CUL3. As shown by the results of CCK- 8 assay, XIST overexpression inhibited cell viability, whereas XIST knockdown increased cell viability. However, CUL3 knockdown abolished the promoting effects of XIST knockdown on cell viability (Fig. 7A). As presented in Fig. 7B and C, XIST overexpression suppressed the differentiation and mineralization of MC3T3-E1 cells. In addition, the promoting effects of XIST silencing on differentiation and mineralization were eliminated by CUL3 knockdown. Finally, the results of western blot analysis and RT-qPCR demonstrated 
A
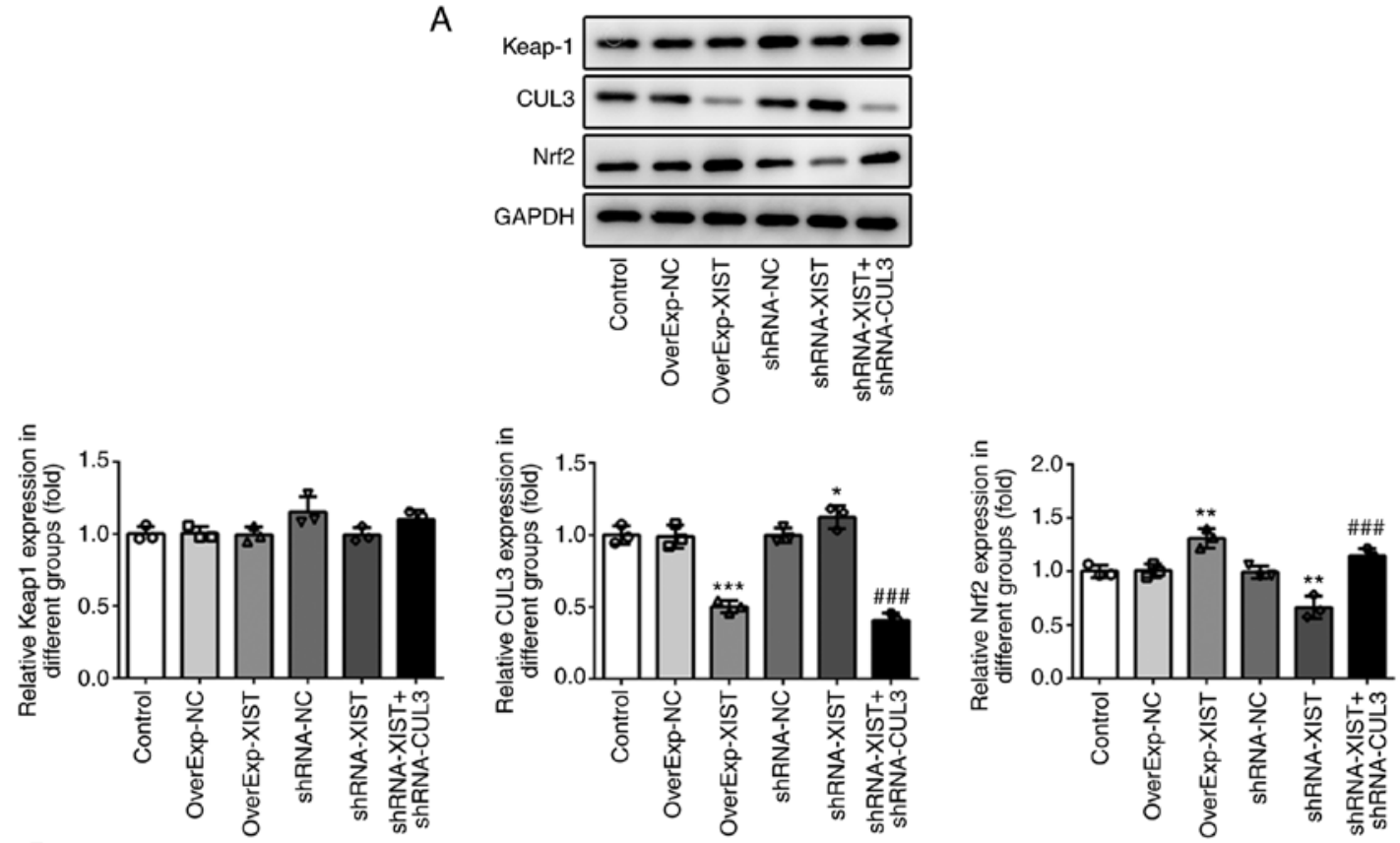

B
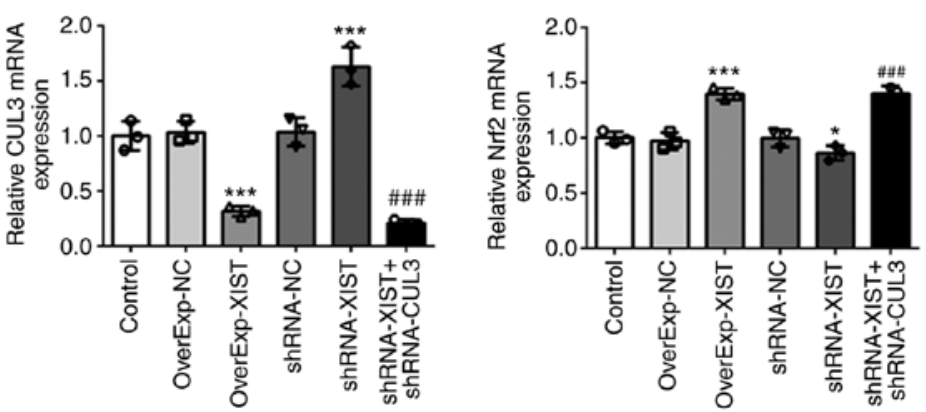

Figure 8. CUL3 downregulation reverses the XIST knockdown-induced effects on the expression levels of Nrf2 and CUL3 in MC3T3-E1 cells. (A and B) The protein and mRNA expression levels of kelch-like ECH-associated protein 1, CUL3 and Nrf2 were detected via western blotting and reverse transcription-quantitative PCR, respectively. Results are presented as the mean $\pm \mathrm{SEM}$ from three independent experiments. ${ }^{*} \mathrm{P}<0.05,{ }^{* * *} \mathrm{P}<0.01,{ }^{* * * *} \mathrm{P}<0.001$ vs. control;

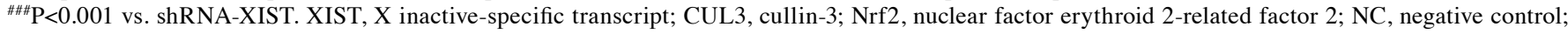
shRNA, short hairpin.

that XIST overexpression significantly downregulated the expression of CUL3 and markedly promoted the expression of Nrf2. By contrast, XIST knockdown notably increased CUL3 expression and markedly decreased Nrf2 expression, which were reversed by CUL3 silencing (Fig. 8A and B). On the whole, these results illustrated that CUL3 knockdown abolished the effects of XIST silencing on the behaviors of MC3T3-E1 cells via Nrf2.

\section{Discussion}

As a chronic skeletal disorder, OP is characterized by damaged bone structure and low bone mass, and can reduce bone strength and an increased susceptibility to fractures (22). It has been reported that estrogen deficiency leads to bone loss via enhancing osteoclastic function (23). Postmenopausal OP is a highly prevalent disease, which contributes to a high morbidity and mortality. Oral bisphosphonates are common therapeutic agents, and the long-term use of these agents can reduce the risk of fractures in patients with OP (24). However, there are various limitations in current drug treatment regimens, including a slow therapeutic effect and an increased risk of adverse events when used over a prolonged period of time. Consequently, the development of novel therapeutic strategies to regulate bone formation in patients with OP is of utmost importance.

A previous study reported that lncRNA XIST is expressed at high levels in the serum and monocytes of patients with OP (15). In the present study, it was observed that XIST overexpression promoted OP by decreasing BMD, the destruction of the cortex and inhibiting bone formation in rats, whereas XIST knockdown inhibited OP progression in rats with OVX-induced OP. Moreover, XIST knockdown enhanced cell viability, as well as the differentiation and mineralization abilities of $\mathrm{H}_{2} \mathrm{O}_{2}$-treated MC3T3-E1 cells. These data indicated that IncRNA XIST enhanced OP development, which was consistent with previous findings (15).

Most importantly, XIST was confirmed to directly target CUL3 and negatively regulate its expression, as determined by a dual-luciferase reporter assay. The Keap1/CUL3/RBX1 complex is a negative regulator of Nrf2 protein expression (12). Under normal conditions, Nrf2 protein interacts with the Keap1/CUL3/RBX1 complex and is maintained at low levels 
due to ubiquitination and proteasomal degradation (25). Under high levels of oxidative stress, the interaction is prevented due to conformational changes of Keap1, resulting in the accumulation of Nrf2 and translocation to the nucleus. Once any component of the complex is absent or altered, Nrf2 is hyperactivated, which leads to its accumulation and translocation into the nucleus (26). In the present study, XIST was confirmed to inhibit CUL3 expression and enhance Nrf2 expression, which was supported by both in vivo and in vitro results. Moreover, XIST suppressed the ubiquitination of Nrf2 in OVX-induced OP rats. Of note, CUL3 knockdown abolished the inhibitory effect of XIST silencing on Nrf2 expression, which indicated that the inhibition of XIST leads to the degradation of Nrf2 by targeting CUL3.

A previous study confirmed that targeting the Keap1/Nrf2 pathway could evidently relieve oxidative stress (27). Furthermore, it is commonly known that ROS play a crucial role in the activation of numerous biological pathways, and are closely associated with cell proliferation, apoptosis and differentiation (28). Estrogen deficiency initiates OP via the overproduction of ROS and hence, antioxidants may be effective therapeutic candidates (29). It has been reported that increased oxidative stress negatively affects bone formation. However, low levels of ROS suppress the mineralization ability of osteoblasts by altering the differentiation of osteoblasts in early stages (30). Hence, Nrf2 hyperactivation may result in low concentrations of ROS, and may thus inhibit the differentiation of osteoblasts. In the present study, the constitutive activation of Nrf2, as a result of CLU3 downregulation via XIST overexpression, suppressed cell viability, differentiation and mineralization of MC3T3-E1 cells. Most importantly, CUL3 downregulation eliminated the suppressive effects of XIST silencing on the cellular behaviors of MC3T3-E1 cells. Taken together, these findings indicated that XIST suppressed the differentiation of osteoblasts and promoted OP via Nrf2 hyperactivation by targeting CUL3. However, the estrous cycle was not analyzed following bilateral ovariectomy, and the atrophy of uterine horns was not evaluated after the animals were sacrificed in the present study, which will be taken into account in future experiments. Additionally, in future studies, the authors aim to determine the role of XIST in oxidative stress and inflammation, in order to provide a more in-depth understanding of the OP pathogenesis.

In conclusion, the present study demonstrated that XIST facilitated OP and suppressed bone formation by regulating the expression of CUL 3 and Nrf2 in rats with OVX-induced OP. In addition, XIST knockdown promoted the survival, differentiation and mineralization of $\mathrm{H}_{2} \mathrm{O}_{2}$-treated MC3T3-E1 cells, which was reversed by CUL3 downregulation. Overall, XIST inhibited the differentiation of osteoblasts and promoted OP by inhibiting the ubiquitination and degradation of $\mathrm{Nrf} 2$ via targeting CUL3.

\section{Acknowledgements}

Not applicable.

\section{Funding}

The present study was supported by the Research Fund for Lin He's Academician Workstation of New Medicine and Clinical Translation in Jining Medical University, China (grant no. JYHL2018FMS12) and Development Project of Medical and Health Technology in Shandong Province, China (grant no. 2018WSB34010).

\section{Availability of data and materials}

The datasets used and/or analyzed during the current study are available from the corresponding author on reasonable request.

\section{Authors' contributions}

$\mathrm{XC}$ and GC designed the experiments and drafted the manuscript. XC, FM, NZ and FG performed the experiments and analyzed the data. XC and GC reviewed the manuscript. All authors read and approved the final manuscript. XC and GC confirm the authenticity of all the raw data.

\section{Ethics approval and consent to participate}

The animal surgical procedures were performed in accordance with the Guidelines for Animal Care and were approved by the Ethics Committee at the Affiliated Hospital of Jining Medical University (Jining, China, no. IACUC-2000509-5).

\section{Patient consent for publication}

Not applicable.

\section{Competing interests}

The authors declare that they have no competing interests.

\section{References}

1. Cosman F, de Beur SJ, LeBoff MS, Lewiecki EM, Tanner B, Randall S and Lindsay R; National Osteoporosis Foundation: Clinician's guide to prevention and treatment of osteoporosis. Osteoporos Int 25: 2359-2381, 2014.

2. Compston JE, McClung MR and Leslie WD: Osteoporosis. Lancet 393: 364-376, 2019.

3. Wu W, Xiao Z, Chen Y, Deng Y, Zeng D, Liu Y, Huang F, Wang J, Bellanti JA, Rong L and Zheng SG: CD39 produced from Human GMSCs regulates the balance of osteoclasts and osteoblasts through the Wnt/ $\beta$-catenin pathway in osteoporosis. Mol Ther 28: 1518-1532, 2020.

4. Eastell R, O'Neill TW, Hofbauer LC, Langdahl B, Reid IR, Gold DT and Cummings SR: Postmenopausal osteoporosis. Nat Rev Dis Primers 2: 16069, 2016.

5. Manolagas SC: Corticosteroids and fractures: A close encounter of the third cell kind. J Bone Miner Res 15: 1001-1005, 2000.

6. Jun JH, Lee SH, Kwak HB, Lee ZH, Seo SB, Woo KM, Ryoo HM, Kim GS and Baek JH: N-acetylcysteine stimulates osteoblastic differentiation of mouse calvarial cells. J Cell Biochem 103: 1246-1255, 2008.

7. Lean JM, Davies JT, Fuller K, Jagger CJ, Kirstein B, Partington GA, Urry ZL and Chambers TJ: A crucial role for thiol antioxidants in estrogen-deficiency bone loss. J Clin Invest 112: 915-923, 2003.

8. Suzuki T, Motohashi H and Yamamoto M: Toward clinical application of the Keap1-Nrf2 pathway. Trends Pharmacol Sci 34: 340-346, 2013.

9. Hyeon S, Lee H, Yang $\mathrm{Y}$ and Jeong W: Nrf2 deficiency induces oxidative stress and promotes RANKL-induced osteoclast differentiation. Free Radic Biol Med 65: 789-799, 2013.

10. Hinoi E, Fujimori S, Wang L, Hojo H, Uno K and Yoneda Y: Nrf2 negatively regulates osteoblast differentiation via interfering with Runx2-dependent transcriptional activation. J Biol Chem 281: 18015-18024, 2006. 
11. Yoshida E, Suzuki T, Morita M, Taguchi K, Tsuchida K, Motohashi H, Doita M and Yamamoto M: Hyperactivation of Nrf2 leads to hypoplasia of bone in vivo. Genes Cells 23: 386-392, 2018.

12. Ziros PG, Manolakou SD, Habeos IG, Lilis I, Chartoumpekis DV, Koika V, Soares P, Kyriazopoulou VE, Scopa CD, Papachristou DJ and Sykiotis GP: Nrf2 is commonly activated in papillary thyroid carcinoma, and it controls antioxidant transcriptional responses and viability of cancer cells. J Clin Endocrinol Metab 98: E1422-E1427, 2013.

13. Fernandes JCR, Acuna SM, Aoki JI, Floeter-Winter LM and Muxel SM: Long Non-Coding RNAs in the regulation of gene expression: Physiology and disease. Noncoding RNA 5: 17, 2019.

14. Ponting CP, Oliver PL and Reik W: Evolution and functions of long noncoding RNAs. Cell 136: 629-641, 2009.

15. Chen X, Yang L, Ge D, Wang W, Yin Z, Yan J, Cao X, Jiang C, Zheng S and Liang B: Long non-coding RNA XIST promotes osteoporosis through inhibiting bone marrow mesenchymal stem cell differentiation. Exp Ther Med 17: 803-811, 2019.

16. Yao Y, Ma J, Xue Y, Wang P, Li Z, Liu J, Chen L, Xi Z, Teng H, Wang Z, et al: Knockdown of long non-coding RNA XIST exerts tumor-suppressive functions in human glioblastoma stem cells by up-regulating miR-152. Cancer Lett 359: 75-86, 2015.

17. Jee WS and Yao W: Overview: Animal models of osteopenia and osteoporosis. J Musculoskelet Neuronal Interact 1: 193-207, 2001.

18. Komori T: Animal models for osteoporosis. Eur J Pharmacol 759: 287-294, 2015

19. Su K, Bai Y, Wang J, Zhang H, Liu H and Ma S: Comparison of hyaluronic acid and PRP intra-articular injection with combined intra-articular and intraosseous PRP injections to treat patients with knee osteoarthritis. Clin Rheumatol 37: 1341-1350, 2018.

20. Aytekin K, Erhan S, Erişgin Z, Esenyel CZ and Takır S: Intra-articular injection of hydrogen sulfide decreased the progression of gonarthrosis. Can J Physiol Pharmacol 97: 47-54, 2019.

21. Livak KJ and Schmittgen TD: Analysis of relative gene expression data using real-time quantitative PCR and the 2(-Delta Delta C(T)) method. Methods 25: 402-408, 2001.
22. Makitie RE, Costantini A, Kampe A, Alm JJ and Makitie O: New insights into monogenic causes of osteoporosis. Front Endocrinol (Lausanne) 10: 70, 2019.

23. Shen G, Ren H, Shang Q, Zhang Z, Zhao W, Yu X, Tang J, Yang Z, Liang D and Jiang X: miR-128 plays a critical role in murine osteoclastogenesis and estrogen deficiency-induced bone loss. Theranostics 10: 4334-4348, 2020.

24. Guiducci L, Vassalle C, Parchi P and Maffei S: Monthly intramuscular neridronate for the treatment of postmenopausal osteoporosis: Results of a 6-year prospective italian study. Int J Endocrinol 2019: 9802827, 2019.

25. Iso T, Suzuki T, Baird L and Yamamoto M: Absolute Amounts and Status of the Nrf2-Keap1-Cul3 complex within cells. Mol Cell Biol 36: 3100-3112, 2016.

26. Martinez VD, Vucic EA, Pikor LA, Thu KL, Hubaux R and Lam WL: Frequent concerted genetic mechanisms disrupt multiple components of the NRF2 inhibitor KEAP1/CUL3/RBX1 E3-ubiquitin ligase complex in thyroid cancer. Mol Cancer 12: 124, 2013

27. Xue Y and Wang AZ: DJ-1 plays a neuroprotective role in SH-SY5Y cells by modulating Nrf2 signaling in response to lidocaine-mediated oxidative stress and apoptosis. Kaohsiung J Med Sci 36: 630-639, 2020.

28. Migliario M, Pittarella P, Fanuli M, Rizzi M and Reno F: Laser-induced osteoblast proliferation is mediated by ROS production. Lasers Med Sci 29: 1463-1467, 2014.

29. Zhou X, Wang Z, Ni Y, Yu Y, Wang G and Chen L: Suppression effect of $\mathrm{N}$-acetylcysteine on bone loss in ovariectomized mice. Am J Transl Res 12: 731-742, 2020.

30. Nicolaije C, Koedam M and van Leeuwen JP: Decreased oxygen tension lowers reactive oxygen species and apoptosis and inhibits osteoblast matrix mineralization through changes in early osteoblast differentiation. J Cell Physiol 227: 1309-1318, 2012.

This work is licensed under a Creative Commons

Attribution-NonCommercial-NoDerivatives 4.0

International (CC BY-NC-ND 4.0) License. 